\title{
Kinderwunschbehandlung in der gynäkologischen Praxis
}

\author{
Christoph Keck, Sabine Segerer
}

\section{Übersicht}

Einleitung

Epidemiologische Aspekte

Anamneseerhebung beim

infertilen Paar

Untersuchung der Frau

Abklärung hormoneller

Störungen

\section{3 \\ 113 \\ 114 \\ 115 \\ 117}

Typische Laborkonstellationen

in der Basisdiagnostik

Polyzystisches Ovar-Syndrom

(PCOS)

\section{Einleitung}

Innerhalb unseres Fachgebietes - der Gynäkologie und Geburtshilfe - gehört die Subspezialität der Reproduktionsmedizin zweifelsohne zu den Bereichen, in denen es in den letzten Jahren zu einer fast dramatischen Entwicklung neuer Behandlungsmöglichkeiten gekommen ist. Somit sind Techniken wie die intrazytoplasmatische Spermieninjektion (ICSI), die testikuläre Spermienextraktion (TESE) oder moderne Verfahren der Kryokonservierungstechnik zu Routinemethoden geworden, die bei entsprechender Indikation jedem Paar mit unerfülltem Kinderwunsch zugänglich sind.

Bei aller Euphorie für diese fortschrittlichen Behandlungsverfahren wird oftmals jedoch vergessen, dass der Frauenarzt vor Ort der erste und wichtigste Ansprechpartner für alle Fragen rund um den Kinderwunsch ist. Nahezu alle diagnostischen Verfahren und ein großer Teil der Behandlungsmethoden bei unerfülltem Kinderwunsch können sehr erfolgreich in der gynäkologischen Praxis durchgeführt werden. Dies beginnt mit der gezielten Anamneseerhebung und gynäkologischen Untersuchung und setzt sich über die Hormonanalytik bis hin zur Prüfung der Eileiterfunktion fort. In vielen gynäkologischen Praxen werden darüber hinaus auch Untersuchungen zur Abklärung des männlichen Sterilitätsfaktors angeboten. Die Palette konservativer Behandlungsmöglichkeiten ist breit und umfasst die Behandlung zugrunde liegender endokrinologischer
Störungen, die Zyklusoptimierung sowie verschiedene Formen der ovariellen Stimulation.

Erst wenn konservative Behandlungsverfahren nicht zum gewünschten Erfolg führen, besteht die Indikation zu reproduktionsmedizinischen Maßnahmen.

Die vorliegende Übersicht soll darstellen, welche Möglichkeiten zur Kinderwunschbehandlung in der gynäkologischen Praxis gegeben sind. Darüber hinaus wird aufgezeigt, in welchen Fällen weiterführende reproduktionsmedizinische Maßnahmen sinnvoll erscheinen und es wird dargestellt, wie die Zusammenarbeit von Frauenarzt und spezialisiertem Reproduktionsmediziner optimal gestaltet werden kann.

\section{Epidemiologische Aspekte}

Eine der wichtigsten Kennzahlen zur Bevölkerungsentwicklung eines Landes stellt die Geburtenrate dar. 2010 wurden in Deutschland 678000 Kinder geboren. Im Jahr 2011 waren es 663000 Kinder - so wenige wie nie zuvor seit dem Zweiten Weltkrieg. Die Geburtenrate pro 1000 Frauen lag in Deutschland im Jahr 2011 bei 8,1; im Vergleich dazu betrug dieser Wert im Jahr 1900 noch 35,8 . 


\section{Definitionen}

\section{Fertilität}

Unter Fertilität versteht man die Fähigkeit, Nachwuchs zu empfangen und auch auszutragen.

\section{Fekundabilität}

Fekundabilität ist definiert als die Wahrscheinlichkeit des Eintretens einer klinischen Schwangerschaft pro Menstruationszyklus.

\section{Sterilität}

Nach der WHO-Definition spricht man von Sterilität, wenn sich bei regelmäßigem ungeschützten Geschlechtsverkehr innerhalb eines Jahres keine Schwangerschaft eingestellt hat [1]. Wenn eine Frau dabei noch nie konzipiert hat, liegt eine primäre Sterilität vor. Wenn bereits eine Schwangerschaft in der Vorgeschichte bestand, handelt es sich um eine sekundäre Sterilität.

\section{Infertilität}

Infertilität beschreibt das Unvermögen, ein lebendes Kind zu gebären.
Fertilitätstherapien zumindest 1-mal in ihrem Leben in Anspruch. Weltweit wird der Anteil der Frauen, die eine Schwangerschaft anstreben und nicht innerhalb von 5 Jahren tatsächlich schwanger werden, auf $1,9 \%$ geschätzt. Der Anteil von Frauen mit bereits einem Kind, die nicht innerhalb von 5 Jahren eine weitere Schwangerschaft erreichen, obwohl sie dies anstreben, wird auf 10,5\% geschätzt [3]. In einem weltweiten geografischen Vergleich waren die Infertilitätsraten in Osteuropa, Nordafrika, dem mittleren Osten, Ozeanien und in Afrika südlich der Sahara am höchsten.

Eine WHO-Studie an mehr als 8500 infertilen Paaren in entwickelten Ländern bezifferte den Anteil der weiblich verursachten Infertilität mit 37\%, den der männlich verursachten Infertilität mit $8 \%$, bei $35 \%$ der Paare wurde sowohl ein weiblicher als auch ein männlicher Faktor identifiziert.

\section{Anamneseerhebung beim infertilen Paar}

Vergleicht man Deutschland mit europäischen Ländern, in denen höhere Geburtenraten erzielt werden, scheint ein Hauptgrund für die geringe Bereitschaft der Frauen, Kinder zu bekommen, in der schlechten Vereinbarkeit von Beruf und Familie zu liegen, gegen den die Frauen in Deutschland ankämpfen müssen. Die Gründe für den dauerhaften negativen Trend der Geburtenrate sind allerdings umstritten und Gegenstand politischer Kontroversen. Die niedrigen und immer weiter absinkenden Geburtenraten in Deutschland sowie der zu beobachtende Trend eines steigenden Durchschnittsalters bei Geburt des 1. Kindes (1991 2011: von 27,3 auf 30,5 Jahre) lassen das Problem der Infertilität und die entsprechenden Maßnahmen der Kinderwunschbehandlung in den Fokus des gesellschaftlichen Diskurses rücken.

Die Geburtenraten in Deutschland zeigen seit Jahren einen negativen Trend.

Für die Beratung einer Patientin bzw. eines Paares mit unerfülltem Kinderwunsch ist es zunächst wichtig, bestimmte Begrifflichkeiten zu klären.

Die Prävalenz der Infertilität betrug laut einer landesweiten US-amerikanischen Studie an mehr als 15000 verheirateten Frauen in den Jahren 1982-2002 zwischen 7,4 und 8,5\% [2]. Im Jahr 2002 konsultierten 2\% der Frauen im reproduktionsfähigen Alter in den USA einen Arzt wegen Fertilitätsproblemen, 10\% nahmen
Wie auch in anderen medizinischen Bereichen beginnt die Diagnostik bei unerfülltem Kinderwunsch mit der gezielten Anamneseerhebung. Die Situation unterscheidet sich jedoch insofern von anderen Fachgebieten, als dass in der Kinderwunschsprechstunde sowohl zur Diagnostik als auch zur Therapie beide Partner Beachtung finden müssen.

\section{Fragebogen vor der eigentlichen Beratung}

Fragen an das Paar. In der Praxis hat es sich bewährt, dem Paar bereits vor dem Beratungsgespräch einen Fragebogen zukommen zu lassen, um relevante Informationen systematisch zu erfassen. Dieser Fragebogen ersetzt natürlich das ausführliche Beratungsgespräch nicht, stellt aber eine gute Vorbereitung auf das eigentliche Gespräch dar und lässt bereits wichtige Rückschlüsse auf mögliche Ursachen des unerfüllten Kinderwunsches zu.

Wichtigste Frage ist die Dauer des Kinderwunsches, da diese bereits die Schwere der Subfertilität/Infertilität des Paares reflektiert.

Fragen an das Paar betreffen weiterhin die Häufigkeit des Geschlechtsverkehrs bzw. Probleme im Zusammenhang mit dem Geschlechtsverkehr (Erektionsstörungen, Orgasmusstörungen etc.). Ebenso wird nach 
vorangegangenen Schwangerschaften und nach dem Ausgang der Schwangerschaften gefragt.

Fragen an den Partner. Auf männlicher Seite wird neben Fragen zur allgemeinen Vorgeschichte v.a. auf besondere Erkrankungen in der Kindheit fokussiert, bzw. auf anamnestische Hinweise auf Deszensusstörungen, Operationen bzw. chronische Erkrankungen. Auch das Thema Genussgifte - Alkohol, Zigaretten etc. - muss aktiv angesprochen werden. Ebenso muss gezielt nach Kindern aus früheren Beziehungen bzw. nach Schwangerschaften und deren Ausgang gefragt werden. Liegen bereits Ergebnisse auswärtiger Fertilitätsuntersuchungen vor, so können diese als Grundlage für die weitere Diskussion herangezogen werden.

Fragen an die Frau. Auf weiblicher Seite müssen neben den allgemeinen Fragen insbesondere Zyklusstörungen erfasst werden, ebenso wie anamnestische Hinweise auf das Vorliegen einer Endometriose, eines Uterus myomatosus, bzw. anderer reproduktionsmedizinisch relevanter Erkrankungen. Zeitpunkt, Verlauf und Ausgang früherer Schwangerschaften finden besondere Beachtung, da sich hieraus unter Umständen auch weitere diagnostische Maßnahmen ergeben.

Gerade auf weiblicher Seite spielen Operationen in der Vorgeschichte eine besondere Rolle. Insbesondere sollte erfragt werden, ob Laparoskopien oder Hysteroskopien mit Prüfung der Tubenfunktion durchgeführt worden sind, da sich hieraus wichtige Informationen für die weitere Behandlung ergeben. Man sollte dann den entsprechenden OP-Bericht anfordern und bewerten.

\section{Tipp für die Praxis}

Wie auch beim Mann müssen ebenso bei der Partnerin die Einnahme von Medikamenten sowie der Konsum von Genussgiften aktiv erfragt und dokumentiert werden.

\section{Untersuchung der Frau}

\section{Körperliche Untersuchung/ gynäkologische Untersuchung}

Bereits aus der allgemeinen körperlichen Untersuchung können sich Hinweise auf mögliche Ursachen des unerfüllten Kinderwunsches ergeben. Während bei untergewichtigen Frauen eine reduzierte Schwangerschaftsrate und erhöhte Abortrate nachzuweisen ist, ist umstritten, ob ein gesteigerter BMI sich generell negativ auf die Implantationsrate auswirkt [4-6].

In beiden Fällen - sowohl bei Adipositas, als auch bei Untergewicht - sollte auch im Hinblick auf mögliche Komplikationen während Schwangerschaft und Geburt als Therapie der ersten Wahl eine Normalisierung des Körpergewichtes angestrebt werden. Es sollte ebenfalls darauf hingewiesen werden, dass insbesondere bei langjährig bestehendem Untergewicht auch nach Normalisierung des Körpergewichtes oftmals eine ovarielle Funktionsstörung bestehen bleibt.

Bei der allgemeinen körperlichen Untersuchung können neben der Erfassung des Körpergewichtes auch Störungen des Behaarungsmusters dokumentiert werden, sodass sich bereits hieraus Hinweise auf das Vorliegen z. B. einer Hyperandrogenämie ergeben können.

\section{Tipp für die Praxis}

Bei Frauen mit unerfülltem Kinderwunsch unterscheidet sich die gynäkologische Untersuchung nicht von der Routineuntersuchung, jedoch sollte gerade bei diesen Frauen auf anatomische Veränderungen geachtet werden.

In seltenen Fällen lassen sich bei der Spekulumeinstellung Doppelanlagen oder Septen darstellen, bzw. narbige Veränderungen oder eine Stenose des Zervikalkanals (z.B. bei Zustand nach Konisation), die als mögliche Infertilitätshindernisse identifiziert werden.

Bei der gynäkologischen Tastuntersuchung können bereits erste Hinweise auf Form- und Größenabweichung des Uterus oder der Adnexe erfasst werden, ebenso ergibt insbesondere die kombiniert rektovaginale Untersuchung zum Teil Hinweise auf Vorliegen einer Endometriose oder eines Uterus myomatosus. 

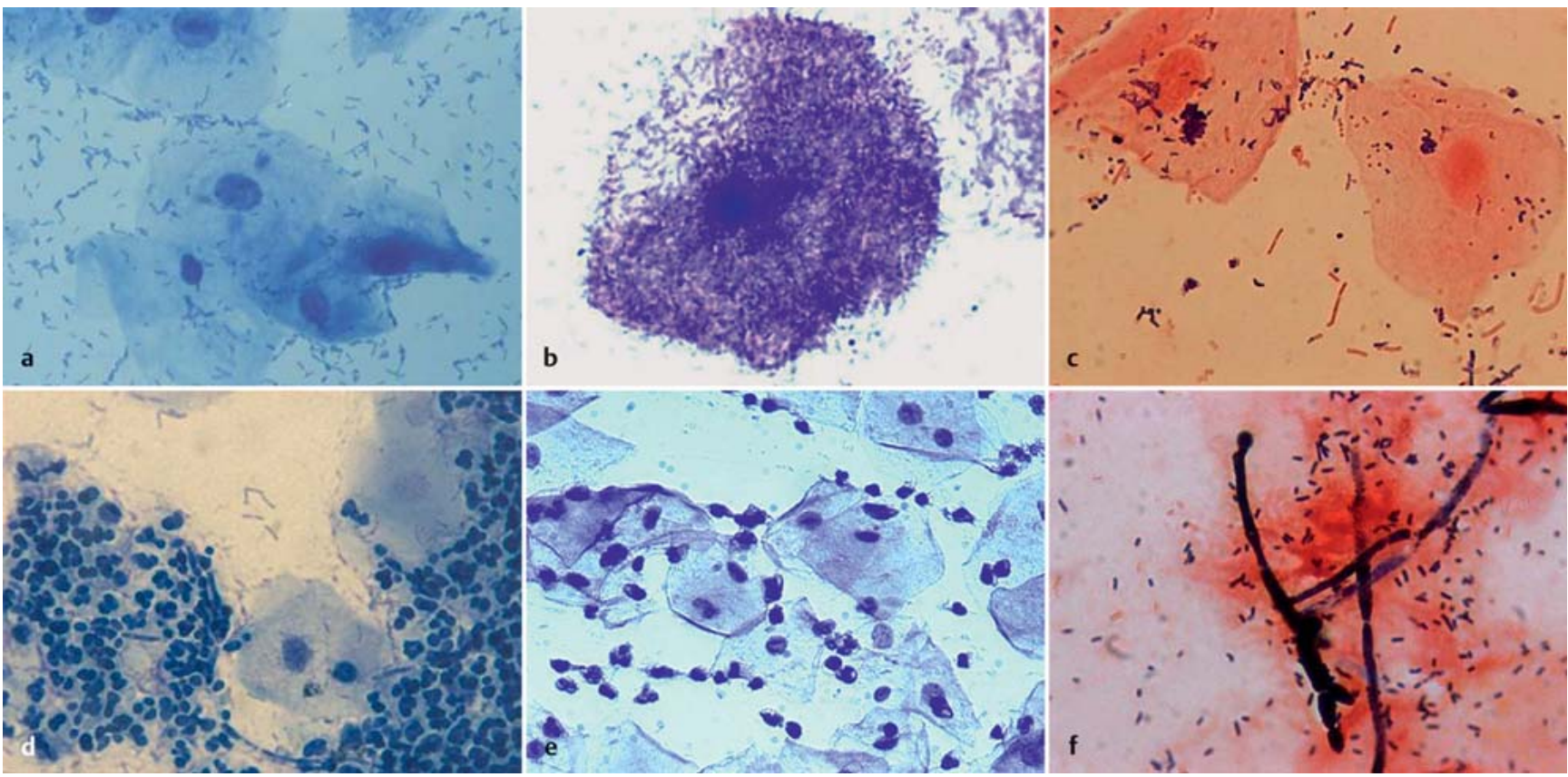

Abb. 1 Typische Befunde im Nativpräparat. a Normalflora. b Clue Cell. c Mischflora. d Massenhaft Leukozyten. e Dysbiose. f Pseudohyphen (Quelle: [17]).

Bei der Erstuntersuchung einer Patientin mit unerfülltem Kinderwunsch sollte geklärt werden, ob ein aktueller PAP-Abstrich vorliegt.

Es wird ein Nativpräparat zum Ausschluss einer aktuellen Infektion und ggf. weitere Abstriche zum Ausschluss einer Chlamydieninfektion entnommen (Abb. 1).

\section{Sonografie}

Uterus. Die Transvaginalsonografie dient - wie sonst auch - unter anderem dazu, die Befunde der Palpationsuntersuchung zu objektivieren und weitere Hinweise auf mögliche Ursachen der Sterilität zu gewinnen. Insbesondere wird dabei noch einmal auf uterine Fehlbildungen bzw. den Ausschluss oder Nachweis von Myomen als typische Fertilitätshindernisse geachtet. Wenngleich die definitive Diagnose einer Endometriose letztlich nur laparoskopisch gestellt werden kann, so können in der transvaginalen Sonografie Hinweise auf Vorliegen einer Adenomyose gewonnen werden, bzw. lässt sich zum Teil die klassische Schokoladenzyste als Hinweis auf eine ovarielle Endometriose darstellen.

Adnexe. Gerade für die Planung des weiteren Vorgehens bei Kinderwunsch muss besonderes Augenmerk auf die Beurteilung der Adnexe gelegt werden, denn nicht nur die Größe des Ovars kann als Hinweis auf eine Funktionseinschränkung herangezogen werden, sondern auch die Anzahl, Größe sowie Anordnung z.B. von Ovarialzysten ist differenzialdiagnostisch wichtig. Ergibt sich das typische Bild einer Hydrosalpinx, so muss bereits primär über die weiterführende laparoskopische Abklärung diskutiert werden. Im Zusammenhang mit der Zyklusanamnese ergibt sich aus der Transvaginalsonografie bereits prima vista eine mögliche Störung der Follikelreifung, sodass die Endometriumsdicke bzw. die Größe und Anzahl follikulärer Strukturen mit der jeweiligen Zyklusphase der Patientin korreliert werden sollte (Abb. 2).

\section{Abklärung des Tubenfaktors: wann und wie?}

Die Abklärung des Tubenfaktors kann mit verschiedenen Untersuchungsmethoden erfolgen, als Goldstandard gilt nach wie vor die Laparoskopie mit Chromopertubation in Kombination mit der Hysteroskopie, insbesondere bei anamnestischen/laborchemischen Hinweisen, wie z.B.:

- Z.n. entzündlichen Erkrankungen

- Tubargravidität in der Vorgeschichte

- operative Eingriffe in der Vorgeschichte

- Z.n. Chlamydieninfektion

- länger andauernde Sterilität ohne sonstige Auffälligkeiten beider Partner 

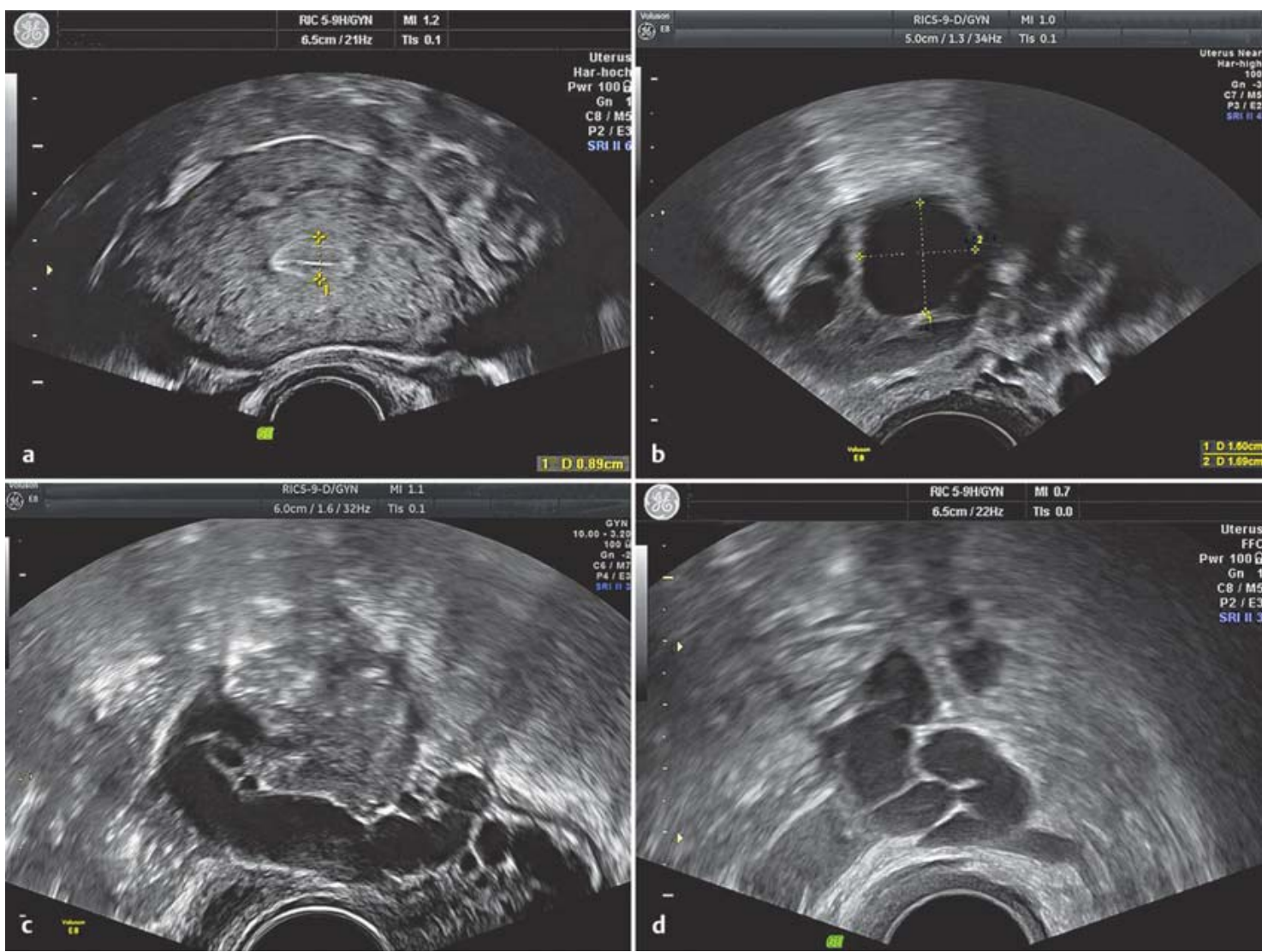

Abb. 2 Transvaginalsonografische Befunde. a Dreischichtiges Endometrium in Zyklusmitte. b Dominanter Follikel. c, d „Posthorn-Phänomen“ bei Sactosalpinx (Quelle: [17]).

Alternativ dazu kommen heute zum Teil weniger invasive Verfahren zum Einsatz, wie. z.B. die Hysterosalpingokontrastsonografie (HSKS) mit einer Sensitivität von 90,4\% und einer Spezifität von 70,3\% [7].

\section{Tipp für die Praxis}

Überlässt man der Patientin mit unerfülltem Kinderwunsch die Wahl des Verfahrens, so wird sie typischerweise - sofern kein Leidensdruck durch akute Beschwerden besteht - das nichtinvasive Verfahren, zum Beispiel die HSKS, präferieren.

Bei einem eingeschränkten Spermiogramm und z.B. geplanten invasiven Therapieverfahren (IVF/ICSI) kann vor Therapiebeginn zunächst auf eine weitere Abklärung verzichtet werden, sofern keine tubaren Risikofaktoren vorliegen. Ebenso kann auf die primäre Abklärung der Tubenfunktion möglicherweise verzichtet werden, wenn andere offensichtliche Ursachen für die Infertilität - z. B. das Vorliegen eines PCO-Syndroms etc. - nachgewiesen wurden und nun primär therapiert werden können.
Nach ausführlicher Beratung muss letztendlich das betroffene Paar bzw. die Patientin die Entscheidung treffen, welches Verfahren zur Abklärung des Tubenfaktors eingesetzt werden soll. Der Vorteil der Laparoskopie mit Hysteroskopie und Chromopertubation liegt zweifelsohne in der zusätzlichen Untersuchungsmöglichkeit intraabdominaler, v.a. auch peritubarer Pathologien (Abb. 3). Ebenso kann in diesem Zusammenhang eine Endometriose definitiv ausgeschlossen werden.

\section{Abklärung hormoneller Störungen}

Prinzipiell kann man bei einem stabilen, regelmäßigen und unauffälligen Zyklus hochgradige endokrinologische Störungen weitestgehend ausschließen. Unabhängig davon können selbstverständlich auch bei regelmäßigem Zyklus Einschränkungen der ovariellen Reserve bzw. Schilddrüsenfunktionsstörungen vorliegen, die durch gezielte endokrinologische Diagnostik erfasst werden müssen. 

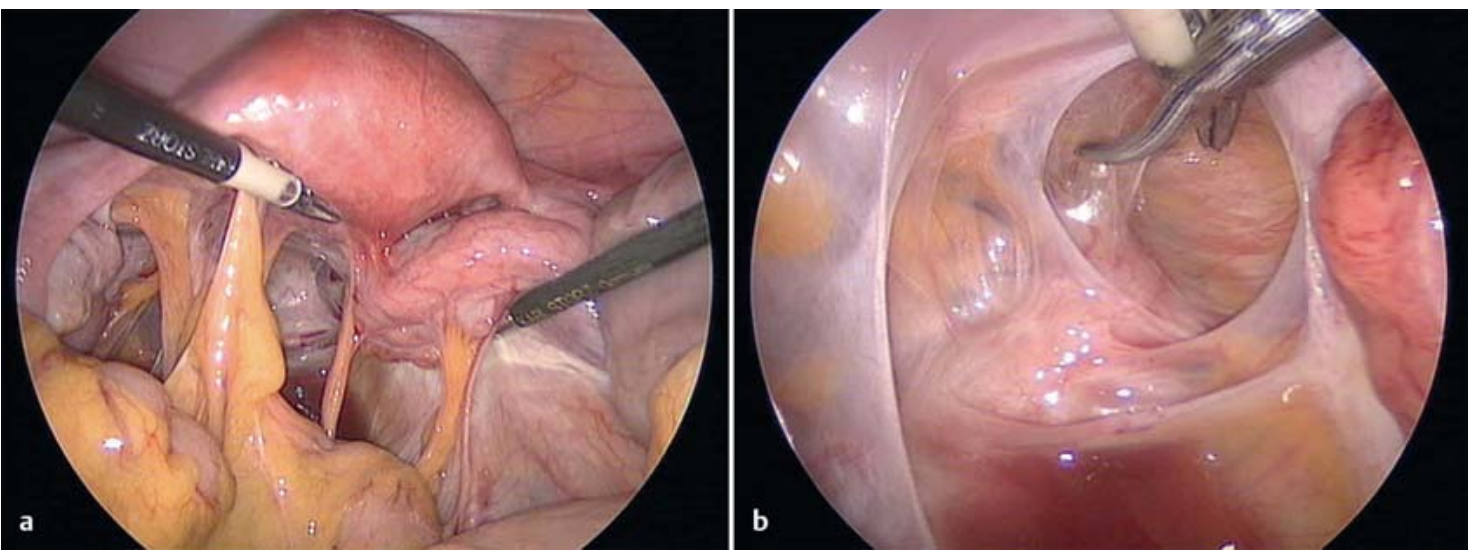

Abb. 3 Adhäsionen nach Infektion mit Funktionseinschränkung der Tuben. a Strangförmige retrouterine Adhäsionen. b Schleierartige intraperitoneale Adhäsionen (Quelle: [17]).

\section{Basisdiagnostik}

Die Basisdiagnostik erfolgt optimal zwischen dem Tag 3 und 5 des Zyklus. Sollen im Zusammenhang mit einem Zyklusmonitoring die weitere Follikelentwicklung und das Konzeptionsoptimum ermittelt werden, so kann eine weitere Untersuchung zwischen Tag 10 und 12 des Zyklus empfohlen werden.

Die Beurteilung der Lutealphase erfolgt typischerweise 6-7 Tage nach stattgehabter Ovulation. Grundsätzlich soll hier jedoch betont werden, dass ein Lutealphasendefizit meist durch prämenstruelles Spotting bzw. Zwischenblutungen in der 2.Zyklusphase auffällig ist. Hormonelle Bestimmungen sind in dieser Hinsicht zweitrangig bzw. können allenfalls als ein Indiz für eine nicht vollwertige Lutealphase gewertet werden.

Zu einer umfassenden frühfollikulären Blutabnahme werden typischerweise folgende Parameter bestimmt:

- Östradiol

- $\mathrm{LH}$

- $\mathrm{FSH}$

- Testosteron

- Dehydroepiandrosteron (DHEAS)

- Androstendion

- Sexualhormon-bindendes Globulin (SHBG)

- Prolaktin

- TSH

In der Zyklusmitte werden im Zusammenhang mit der Ultraschalluntersuchung Östradiol und LH bestimmt sowie in der Lutealphase Östradiol und Progesteron.

\section{Bestimmung des Anti-Müller-Hormons (AMH)}

Je nach Ausgangssituation kann bereits in der Basisuntersuchung AMH zusätzlich bestimmt werden. AMH wird in den Granulosazellen v.a. der Primär-, Sekundär- und frühen Antralfollikel gebildet. AMH wird häufig als Marker der ovariellen Reserve eingesetzt. Dies ist grundsätzlich sinnvoll und richtig, jedoch müssen bei der Interpretation der AMH-Werte die anamnestischen Angaben der Patientin berücksichtigt werden [8]. Ebenso muss beachtet werden, dass AMH zwar eine Abschätzung der Follikelanzahl, jedoch nicht der Qualität der Eizellen erlaubt.

Aus einer einzelnen AMH-Bestimmung lässt sich nicht vorhersagen, ob und wenn ja mit welcher Wahrscheinlichkeit eine Schwangerschaft eintreten wird. Die klinische Relevanz der AMH-Bestimmung zeigt sich v.a. in folgenden Situationen:

- Bei einer auffälligen Konstellation von Östradiol und FSH zwischen Tag 3 und 5 erlaubt die zusätzliche Bestimmung von AMH eine Einschätzung der ovariellen Reserve und eine Abgrenzung gegen zufällige, irrelevante Veränderungen der im Zyklus stark schwankenden Werte von Östradiol und FSH.

- AMH erlaubt bei der ovariellen Stimulation mit Gonadotropinen - neben anderen Faktoren - die Einschätzung der notwendigen FSH-Dosis. Dadurch kann eine mangelhafte Antwort der Ovarien ebenso vermieden werden wie eine überschießende Antwort und das Risiko eines ovariellen Überstimulationssyndroms im Rahmen einer IVF. 


\section{Tipp für die Praxis}

Wenngleich die AMH-Serum-Konzentration - verglichen mit FSH - vergleichsweise konstanter im Zyklusverlauf ist, so sollte $\mathrm{AMH}$ ebenso in der Follikelphase gemessen werden. Postovulatorisch, unter Einnahme kombinierter Kontrazeptiva sowie in der Schwangerschaft liegt der Wert etwa $30 \%$ niedriger als in der Follikelphase eines hormonell nicht beeinflussten Zyklus [8].

\section{Typische Laborkonstellationen in der Basisdiagnostik}

Im Folgenden werden typische Laborkonstellationen vorgestellt, die sich in Abweichung vom physiologischen Hormonprofil bereits in der Basisuntersuchung ergeben können (s. Tab. 1).

\section{Hyperprolaktinämie}

Die Hyperprolaktinämie gehört zu den häufigsten Ursachen von Zyklusstörungen, daher gehört die Prolaktinbestimmung auch zur Basisdiagnostik. Vor Durchführung einer weiterreichenden Diagnostik sollte hier eine Schilddrüsendysfunktion als Ursache der Prolaktinerhöhung, aber auch die Einnahme von Prolaktin- steigernden Medikamenten (insbesondere Psychopharmaka), ausgeschlossen werden.

In vielen Fällen liegt eine rein funktionelle Hyperprolaktinämie vor oder es besteht eine verstärkte Prolaktinausschüttung als Folge eines Mikro- oder Makroprolaktinoms. Makroprolaktinome sind eher selten und fallen meist durch klinische Symptome wie Kopfschmerzen und Sehstörungen auf, während Mikroprolaktinome neurologisch meist asymptomatisch sind und nach endokrinologischer Diagnostik durch gezielte kernspintomografische Untersuchung dargestellt werden.

Die Behandlung der Hyperprolaktinämie erfolgt heute nahezu ausschließlich medikamentös durch Gabe von Dopaminagonisten.

\section{Schilddrüsenfunktionsstörungen}

Schilddrüsenfunktionsstörungen sind häufig und betreffen Frauen etwa 10-mal häufiger als Männer. In Deutschland sind mehr als $11 \%$ aller Menschen von einer Funktionsstörung der Schilddrüse betroffen.

Bei einer Funktionsstörung der Schilddrüse sind die latenten Formen, bei denen die Schilddrüsenhormone noch normal, jedoch das TSH zu hoch (latente Hypothyreose) oder zu niedrig ist (latente Hyperthyreose), bis zu 10-mal häufiger als die manifesten Formen.

\section{Tabelle 1}

Hormonelle Störungen mit Abweichungen vom physiologischen Hormon-Basisprofil.

\begin{tabular}{|c|c|c|}
\hline Art der Störung & Ursache & Laborauffälligkeit \\
\hline $\begin{array}{l}\text { hypogonadotroper } \\
\text { Hypogonadismus }\end{array}$ & $\begin{array}{l}\text { - angeboren (z. B. Kallmann-Syndrom) } \\
\text { - erworben (z. B. Hypophyseninfarkt } \\
\text { im Rahmen des Sheehan-Syndroms) } \\
\text { - iatrogen (Radiatio) }\end{array}$ & $\begin{array}{l}\text { - niedrige Gonadotropine } \\
\text { - ggf. weiterführende Diagnostik: } \\
\text { GnRH-Stimulationstest, cMRT }\end{array}$ \\
\hline $\begin{array}{l}\text { hypothalamo-hypophysäre } \\
\text { Dysfunktion }\end{array}$ & $\begin{array}{l}\text { - Hyperandrogenämie } \\
\text { (inkl. Hypercortisolismus) } \\
\text { - Essstörungen, Leistungssport, Stress } \\
\text { - Hyperprolaktinämie } \\
\text { - Schilddrüsenfunktionsstörungen } \\
\text { - idiopathisch }\end{array}$ & - LH kann erhöht, niedrig oder normal sein \\
\hline $\begin{array}{l}\text { hypergonadotroper } \\
\text { Hypogonadismus }\end{array}$ & $\begin{array}{l}\text { - idiopathisch } \\
\text { - iatrogen (postoperativ, Radiatio, Chemotherapie) } \\
\text { - autoimmun }\end{array}$ & - hohe Gonadotropine \\
\hline
\end{tabular}




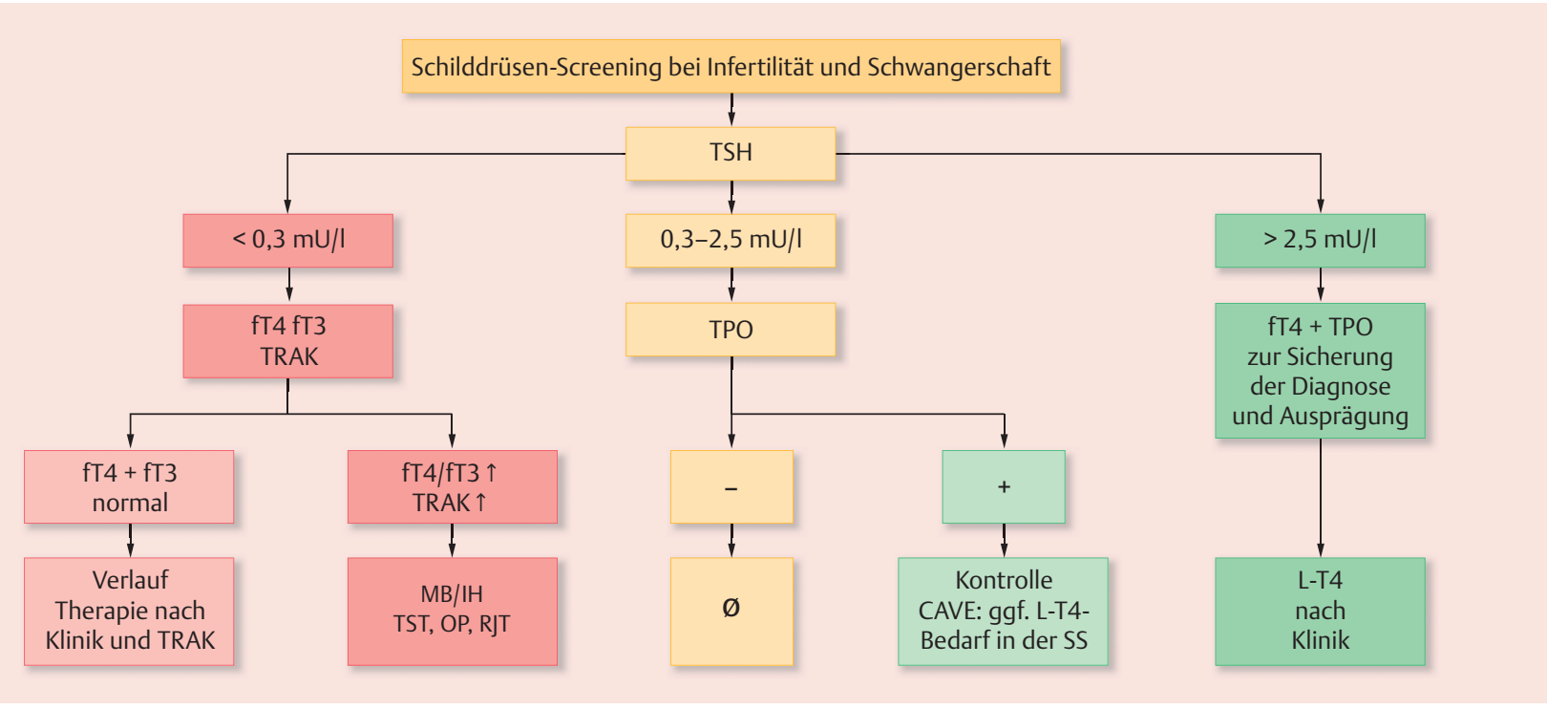

Abb. 4 Schilddrüsen-Screening bei Infertilität und Schwangerschaft (IH: immunogene Hyperthyreose; L-T4: Substitution mit L-Thyroxin; MB: Morbus Basedow; OP: Operation; RJT: Radiojodtherapie; SS: Schwangerschaft; TPO: Thyreoperoxidase-Antikörper; TRAK: TSH-Rezeptor-Antikörper, TSH: Thyreoidea-stimulierendes Hormon; TST: thyreostatische Therapie) (Quelle: [17]).

Bei unerfülltem Kinderwunsch lässt sich in vielen Fällen eine Hypothyreose bei Autoimmunthyreoiditis (AIT) nachweisen, die unbehandelt nicht nur die Fertilität beeinträchtigt, sondern v.a. in der Schwangerschaft zu erheblichen Komplikationen beim Kind führen kann. Auch beim PCOS findet sich überzufällig häufig eine AIT, die die damit assoziierte Infertilität damit noch weiter aggraviert.

Zur Basisdiagnostik wird üblicherweise TSH bestimmt, ggf. bereits im Screening ergänzt um TPO-Antikörper. Bei der weiterführenden Diagnostik werden üblicherweise parallel TSH, fT4, fT3, TPO-Antikörper und TSH-Rezeptor-Antikörper (TRAK) bestimmt (Abb. 4).

Unter Euthyreose versteht man den Nachweis eines normalen basalen TSH-Spiegels und normaler Werte für das fT4 sowie das fT3. Bei normalen peripheren Schilddrüsenhormonspiegeln, jedoch erniedrigtem bzw. erhöhtem TSH-Spiegel (latente Hyper- bzw. Hypothyreose) ist eine weiterführende Diagnostik erforderlich. Hier müssen folgende Erkrankungen ausgeschlossen werden:

- immunogene Hypothyreose

- Morbus Basedow

- Schilddrüsenautonomie

Hypothyreose. Für die Patientin mit unerfülltem Kinderwunsch sollte bereits präkonzeptionell ein TSH-Wert $<2,5$ angestrebt werden. Dies gelingt üblicherweise durch Einstellung mit täglichen Gaben von
L-Thyroxin morgens oral, vor dem Frühstück. Die Dosis ist individuell anzupassen. Bei latenter Hypothyreose liegt die erforderliche Tagesdosis meist bei $50-100 \mu \mathrm{g}$ L-Thyroxin. Keine Indikation zur L-Thyroxingabe besteht bei positivem Antikörpernachweis, aber euthyreoter Stoffwechsellage. Zusätzlich zur L-Thyroxinsubstitution bei Euthyreose - auch ohne eine AIT - wird eine Substitution mit Folsäure, Vitamin $B_{12}$ und Jodid $(150-200 \mu \mathrm{g} / \mathrm{d})$ empfohlen. Eine immunsuppressive Therapie ist bei AIT kontraindiziert, eine Operationsindikation besteht nur bei konkretem Malignomverdacht.

Bei allen Patientinnen mit nachgewiesener AIT sind lebenslange Kontrolluntersuchungen in mindestens jährlichem Abstand sinnvoll.

Bei Kinderwunschpatientinnen sollte eine Überprüfung der Schilddrüsenfunktion alle 3 Monate erfolgen, in der Schwangerschaft alle 4-6 Wochen.

Hyperthyreose. Im Gegensatz zur Hypothyreose stellt die Hyperthyreose sich eher selten dar. Als häufigste Ursache muss an eine Hyperthyreose im Zusammenhang mit dem Morbus Basedow gedacht werden. Sie kann entgegen dem klassischen Krankheitsbild auch ohne endokrine Orbitopathie und sogar ohne Struma auftreten und wird dann immunogene Hyperthyreose genannt. Verursacht wird die Hyperthyreose durch die TSH-ähnliche Wirkung von Autoantikörpern gegen den TSH-Rezeptor (TRAK). Die Prävalenz für den Morbus 
Basedow liegt bei 0,1-0,2\%. Frauen sind 5- bis 10-mal häufiger davon betroffen als Männer.

Der Beleg der Hyperthyreose gelingt durch den Nachweis eines vollständig supprimierten TSH-Spiegels und erhöhter Werte für fT3 und fT4. Bei über 90\% der Patienten lassen sich TRAK-Antikörper nachweisen, in etwa $70 \%$ der Fälle auch die TPO-Antikörper.

Eine manifeste Hyperthyreose muss immer behandelt werden. Meist besteht auch bei latenter Hyperthyreose eine Therapieindikation. Primär wird ein medikamentöser konservativer Therapieversuch mit Thyreostatika über 12-15 Monate durchgeführt. Hierzu stehen Thiamazol, Carbimazol und Propylthiouracil zur Verfügung, dabei sollte man bei Frauen mit Kinderwunsch Propylthiouracil den Vorzug geben. Eine Operation oder Radiojodtherapie erfolgt bei Rezidiv der Erkrankung, wenn ein schnelleres Erreichen einer Euthyreose erforderlich ist, oder wenn andere Gründe gegen eine primär medikamentöse Therapie sprechen.

Bei manifester Hyperthyreose ist eine Jodidgabe kontraindiziert.

\section{Tipp für die Praxis}

Stellt der behandelnde Frauenarzt bei einer Patientin mit unerfülltem Kinderwunsch in der Basisdiagnostik oder bei der erweiterten Diagnostik eine Schilddrüsenfunktionsstörung fest, sollte diese in enger Kooperation mit entsprechend erfahrenen Endokrinologen behandelt werden.

Eine Kinderwunschtherapie sollte erst dann vorgenommen werden, wenn eine euthyreote Stoffwechsellage erzielt wurde.

\section{Hyperandrogenämie}

Die Hyperandrogenämie gehört zu den häufigsten Störungen der weiblichen Fertilität. Wir unterscheiden dabei zwischen einer primär ovariell bedingten bzw. adrenalen Form der Hyperandrogenämie. Hierzu dient die Bestimmung von:

- Testosteron

- DHEAS

- Androstendion

- SHBG

- FAI (freier Androgen-Index)

Adrenale Hyperandrogenämie. Eine adrenal bedingte Hyperandrogenämie lässt sich oftmals an einer erhöhten Serumkonzentration für DHEAS oder Androsten- dion nachweisen. In diesen Fällen sollte frühfollikulär zusätzlich 17-Hydroxyprogesteron (17-OHP) bestimmt werden als Marker für das Vorliegen eines Late-OnsetAGS. Dies hat Konsequenzen für die weitere Diagnostik (ACTH-Stimulationstest und ggf. molekulargenetische Untersuchung). Ebenfalls wäre bei einer Erhöhung der adrenalen Androgene eine Überprüfung des basalen Cortisolspiegels sinnvoll, bei Erhöhung ggf. ein Dexamethason-Hemmtest, um ein Cushing-Syndrom ausschließen zu können.

Die ovarielle Hyperandrogenämie ist weitaus häufiger anzutreffen als die adrenale Form.

Ovarielle Hyperandrogenämie. Bei überwiegend ovariell bedingter Hyperandrogenämie liegt meist ein erhöhter Testosteronspiegel vor. Durch Bestimmung der SHBG-Konzentration sowie durch die Berechnung des FAI gelingt eine Abschätzung des Anteils des freien und damit biologisch wirksamen Testosterons. Die häufigste Ursache für eine ovarielle Hyperandrogenämie wiederum stellt das Syndrom der polyzystischen Ovarien (PCOS) dar (Abb. 5). Dieses wird im folgenden Abschnitt gesondert beschrieben.

\section{Polyzystisches Ovar-Syndrom (PCOS)}

Die Ursache des PCOS ist unbekannt, die klinischen, sonografischen und biochemischen Charakteristika der Erkrankung treten in verschiedenen Kombinationen und mit unterschiedlicher Ausprägung auf.

Symptomatik. Es existieren zum Teil divergierende Definitionen des PCOS, meist wird heute jedoch die Klassifikation nach den Rotterdam-Kriterien vorgenommen. Danach kann die Diagnose PCOS gestellt werden, wenn mindestens 2 der folgenden Symptome vorliegen:

- Oligo-/oder Anovulation

- klinische und/oder biochemische Zeichen der Hyperandrogenämie

- sonografisches Bild der polyzystischen Ovarien, definiert als mindestens 12 Follikel mit max. $10 \mathrm{~mm}$ Durchmesser in mindestens einem Ovar (s.Abb. 6)

Die typischen Symptome des PCOS sind Zyklusstörungen, Hyperandrogenismus und polyzystische Ovarien. In der Praxis zeigt sich, dass es bei Frauen, die normalgewichtig bzw. sogar schlank sind und keine Symptome des Hyperandrogenismus aufweisen, bisweilen gerau- 


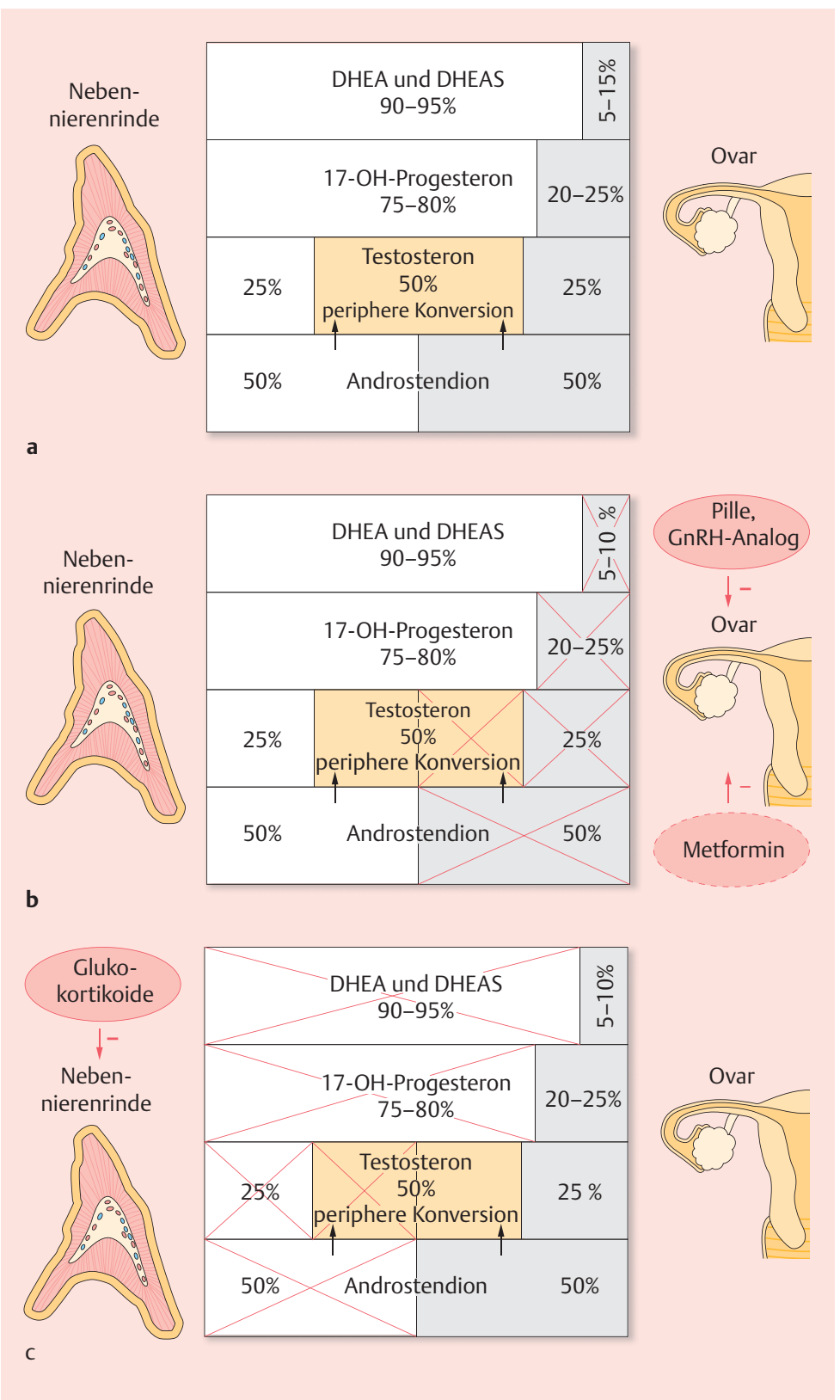

Abb. 5 Androgene der Frau. a Hauptandrogene der Frau und deren Herkunft aus Nebennierenrinde, Ovar und peripherem Metabolismus. Das 17-OH-Progesteron hat selbst keine androgene Wirkung und ist als diagnostisch sehr wichtige Androgenvorstufe der Nebenniere aufgeführt. b Möglichkeiten der therapeutischen Suppression der ovariellen Androgene. Bei Insulinresistenz ist Metformin eine therapeutische Option. c Therapeutische Suppression der andrenalen Androgene (Quelle: [17]). me Zeit dauert, bis die ovarielle Funktionsstörung eindeutig dem Formenkreis des PCOS zugeordnet wird.

\section{Therapie des PCOS}

Da die Ursache des PCOS unbekannt ist, steht definitionsgemäß keine kausale Therapie zur Verfügung.

Die Behandlung richtet sich nach dem Therapieziel und dem individuell vorliegenden Beschwerdekomplex.

Sofern kein Kinderwunsch besteht und das Hauptziel in der Linderung hyperandrogenämischer Symptome liegt, kommt für junge Frauen z.B. die Verordnung eines antiandrogen wirksamen Kontrazeptivums infrage.

Stehen die metabolischen Aspekte und die Adipositas im Zentrum der therapeutischen Bemühungen, muss über eine Maßnahme der Gewichtsreduktion (Ernährungsberatung, Diät, Sport etc.), den Einsatz von Medikamenten (Metformin, Orlistat, Liraglutid etc.) und in extremen Fällen auch über eine bariatrische Operation diskutiert werden.

Bei Frauen mit PCOS und Kinderwunsch besteht das primäre Behandlungsziel darin, die ovarielle Funktion zu verbessern und einen regelrechten ovulatorischen Zyklus zu etablieren. Hierzu kommen - abhängig von der Ausgangssituation - folgende Maßnahmen zum Einsatz:

- Gewichtsabnahme und Lebensstil-Modifikation bei übergewichtigen Patientinnen

- Clomifen/Gonadotropine zur Induktion einer Follikelreifung

- Metformin zur Behandlung der Insulinresistenz

- Ovarian Drilling

\section{Gewichtsabnahme}

Der Anteil adipöser Frauen mit PCOS wird in den Studien mit 30-75\% angegeben. Meist liegt eine androide Fettverteilung vor. In der überwiegenden Zahl der Studien konnte gezeigt werden, dass eine Reduktion des Körpergewichts um 5\% zu einer signifikanten Verbesserung der Zyklizität, des Hirsutismus und zu einer entsprechenden Abnahme der Serumspiegel für Insulin, Androstendion, DHEAS, Testosteron sowie des LH/ FSH-Quotienten führt. Die verbesserte Zyklizität geht dann typischerweise auch mit ovulatorischen Zyklen 


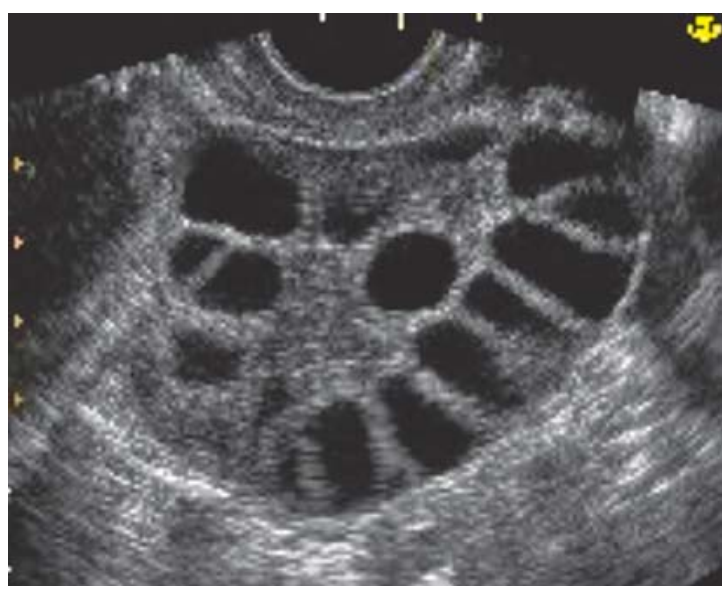

Abb. 6 Polyzystisches Ovar mit typischem „Perlschnur-Phänomen“, d. h. multiplen, randständigen Follikeln (Quelle: [17]).

und dementsprechend verbesserten Konzeptionschancen einher [9].

Bei adipösen Frauen mit PCOS besteht die Therapie der ersten Wahl in der Gewichtsreduktion.

\section{Tipp für die Praxis}

Der Frauenarzt sollte adipöse Patientinnen bezüglich der Gewichtsreduktion entsprechend beraten bzw. ein Netzwerk etablieren, in dem sich z. B. Ernährungsberater, Sportmediziner etc. um diese Frauen kümmern.

\section{Clomifenstimulation}

Clomifen gehört zu den häufigsten in der gynäkologischen Praxis eingesetzten Medikamenten. Die Substanz ist seit den 1960er-Jahren in Gebrauch. Es handelt sich dabei um einen Östrogenrezeptor-Modulator (SERM), der im Hypothalamus zu einer Blockade von Östrogenrezeptoren führt. Dies bewirkt wiederum eine Steigerung der hypophysären FSH- und LH-Ausschüttung und damit eine Stimulation der Eizellreifung im Ovar.

Clomifen kann - je nach Rezeptorausstattung des Zielgewebes - an bestimmten Organen auch antiöstrogene Wirkung entfalten. So kommt es unter dem Einfluss von Clomifen bei einigen Patientinnen zu einem inadäquaten Aufbau des Endometriums bzw. zu einer deutlichen Zunahme der Viskosität des Zervixschleims, was sich negativ auf die Konzeptionschancen auswirken kann.
Vor Einleitung der Therapie muss die medizinische Indikation geprüft werden.

Typischerweise wird Clomifen eingesetzt, um bei Frauen mit hypothalamisch-hypophysärer Dysfunktion eine Follikelreifung zu induzieren. Unabhängig davon liegt ein weiteres Einsatzgebiet für Clomifen in der Stimulation vor intrauteriner Insemination (Abb. 7).

Dosierung. Clomifen wird meist in einer Dosierung von $50 \mathrm{mg} / \mathrm{d}$ über 5 Tage verabreicht. Der Therapiebeginn liegt üblicherweise zwischen dem 2. und 5. Zyklustag (somit erfolgt eine Stimulation z. B. vom 2.-6. oder 3. -7., 4.-8. oder 5.-9. Zyklustag).

Bei amenorrhoischen Patientinnen kann versucht werden, aus der bestehenden Amenorrhö heraus Clomifen zu verabreichen, um damit eine Follikelreifung herbeizuführen. Voraussetzung ist eine Endometriumdicke $<5-6 \mathrm{~mm}$ sowie das Fehlen von Follikeln $>10 \mathrm{~mm}$. Ansonsten sollte zuvor eine Abbruchblutung z.B. durch eine zyklische Gestagengabe in Transformationsdosis erfolgen.

Bei dem o.g. Vorgehen kommt es in etwa der Hälfte der Fälle zur Follikelreifung und zu ovulatorischen Zyklen. Bei den Frauen, die auf 50 mg nicht adäquat reagieren, kann die Dosis in einem Folgezyklus auf 100 bzw. $150 \mathrm{mg} / \mathrm{d}$ - ebenfalls über einen Zeitraum von 5 Tagen verabreicht - erhöht werden. Dabei muss beachtet werden, dass nur ca. 20\% der Frauen, die auf $50 \mathrm{mg}$ Clomifen nicht reagieren, mit $100 \mathrm{mg}$ adäquat stimuliert werden können (s. Tab. 2). Frauen, die auch auf $100 \mathrm{mg}$ Clomifen nicht reagieren, zeigen bei einer Dosiserhöhung auf $150 \mathrm{mg}$ lediglich in 8-9\% der Fälle eine entsprechende ovarielle Reaktion [10]. Insofern ist ein Versuch mit $100 \mathrm{mg}$ nach Scheitern von $50 \mathrm{mg}$ zu überlegen. Aussichtsreich erscheint das, wenn es auf $50 \mathrm{mg}$ zumindest eine geringe Reaktion gegeben hat. Die nachfolgende Steigerung auf $150 \mathrm{mg}$ halten wir bei

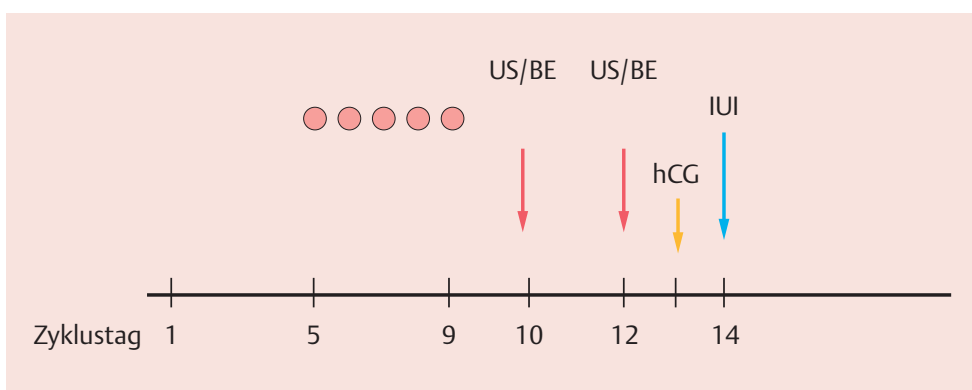

Abb. 7 Schematischer Ablauf der Clomifenstimulation (BE: Blutentnahme zur Hormonbestimmung; IUI: intrauterine Insemination; US: Ultraschalluntersuchung) (Quelle: [17]). 


\section{Tabelle 2}

Ovulationsraten bei Gabe von 50, 100, 150 mg Clomifen (Metaanalyse von 13 Studien; nach [10]).

\begin{tabular}{ll} 
Dosierung (mg) & Ovulationsrate \\
\hline 50 & $46 \%$ \\
\hline 100 & $21 \%$ (der „Versager“ mit $50 \mathrm{mg})$ \\
\hline 150 & $8 \%$ (der „Versager“ mit $100 \mathrm{mg})$ \\
\hline
\end{tabular}

einer Chance von $<10 \%$ für eine ovarielle Reaktion nicht für sinnvoll.

Ovulationsinduktion durch hCG. In den meisten Fällen kommt es nach Clomifenstimulation zwar zu einer spontanen Ovulation, jedoch ist das Zeitfenster relativ weit, innerhalb dessen sich die Ovulation ereignet. So konnten Ovulationen ab einer Follikelgröße von 14$15 \mathrm{~mm}$ nachgewiesen werden. In manchen Fällen kam es jedoch erst ab einem Follikeldurchmesser $>25 \mathrm{~mm}$ zur Spontanovulation. Dies macht es schwierig, dem Paar den idealen Zeitpunkt zum Geschlechtsverkehr zu nennen, ebenso wird dadurch z. B. die Planbarkeit einer intrauterinen Insemination erschwert. Aus diesem Grund kann eine Clomifenstimulation mit der gezielten Gabe von hCG zur Ovulationsinduktion kombiniert werden. Hierzu werden üblicherweise 5000 Einheiten hCG verabreicht. Dann kommt es ca. 36-40 h später zur Ovulation.

Eine signifikante Verbesserung der Schwangerschaftsrate konnte durch zusätzliche hCG-Gabe bisher nicht hinreichend belegt werden.

\section{Tipp für die Praxis}

Der optimale Zeitpunkt, um eine Schwangerschaft zu induzieren, liegt 36-48 h vor der Ovulation - insofern sollte der Geschlechtsverkehr bei Gabe von hCG zeitgleich dazu stattfinden. „Zeitgleich“ bedeutet dabei einen Zeitraum von $\pm 2-4 \mathrm{~h}$ vor oder nach Gabe von hCG. Die wiederholte Kohabitation erreicht dabei keine höheren Schwangerschaftsraten als die einmalige.

Zusätzliche Gestagengabe? Ein routinemäßiger Support der Lutealphase ist nach Clomifenstimulation nicht erforderlich; bei monofollikulärer Reifung und konsekutiver Ovulation kann durch routinemäßige Gestagengabe in der Lutealphase kein Benefit erzielt werden. Es wird diskutiert, ob eine zusätzliche Gestagengabe sinnvoll erscheint, sofern 2 oder gar mehr Follikel unter Clomifen heranreifen. Im Falle einer bi- oder polyfollikulären Reifung muss man davon ausgehen, dass die höheren Steroidhormon-Konzentrationen über eine negative Rückkoppelung zu einer mangelhaften Lutealphase führen, sodass dann eben die Lutealphasen-Unterstützung sinnvoll wird und die Schwangerschaftschancen sich optimieren lassen.

Nebenwirkungen unter Clomifen. Zu den allgemeinen Nebenwirkungen von Clomifen gehören:

- Übelkeit (bis 5\%)

- Sehstörungen $(<2 \%)$

- erhöhte Sensibilität der Mammae

- Unterbauchschmerzen

Die Inzidenz von Mehrlingsschwangerschaften wird in der Literatur mit 10-15\% angegeben.

Eine klinisch relevante ovarielle Überstimulation dürfte unter Clomifen eher selten auftreten, in der Weltliteratur existiert lediglich eine Handvoll Kasuistiken dazu. Die in der gynäkologischen Praxis am häufigsten dokumentierte Nebenwirkung von Clomifen liegt im partiell antiöstrogenen Effekt der Substanz auf den Zervixschleim bzw. auf das Endometrium (Abb. 8).

So zeigen ca. 15\% der Patientinnen trotz guter ovarieller Reaktion einen ungünstigen Zervix-Score bzw. einen inadäquaten Endometriumaufbau. In dieser Situation kann versucht werden, durch flankierende Gabe von Östradiol (2 -6 mg/d ab Zyklusbeginn) den antiöstrogenen Effekt von Clomifen zu kompensieren. Es ist allerdings bis heute unklar, ob sich daraus höhere Konzeptionschancen ableiten lassen. Da Clomifen die Östrogenrezeptoren blockiert, wird auch die zusätzliche Gabe von Östradiol nur bedingt Wirkung entfalten können.

\section{Tipp für die Praxis}

Wir führen nach Clomifenstimulation bei einem auffälligen Zervix-Score keine flankierende Östrogenisierung durch, sondern empfehlen eher das Umschwenken auf eine Gonadotropinstimulation.

Zu den ansonsten relevanten Nebenwirkungen des Clomifens muss auch die erhöhte Inzidenz von Mehrlingsschwangerschaften gezählt werden. Es konnte gezeigt werden, dass in den USA 22\% aller Zwillingssowie $40 \%$ aller Drillings- und $71 \%$ aller Vierlings- oder noch höhergradigen Mehrlingsschwangerschaften auf 

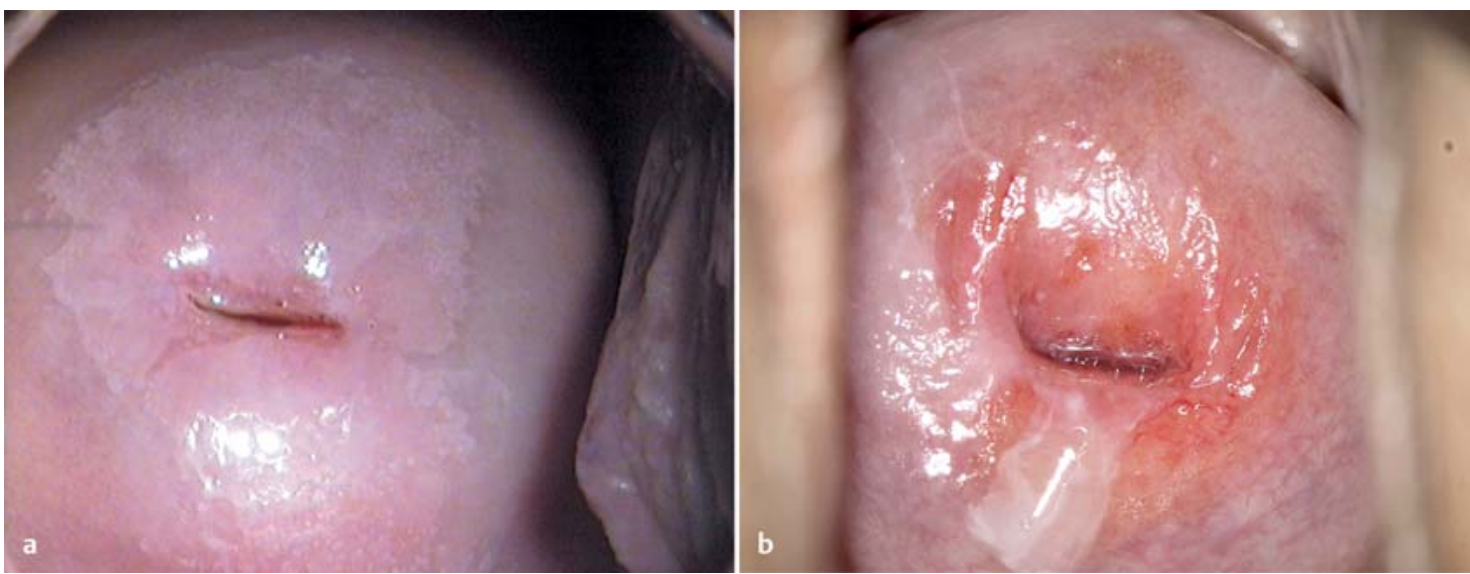

Abb. 8 Zervix-Score: Nebenwirkung von Clomifen. a Antiöstrogener Effekt bei ca. 15\% der Patientinnen, erkennbar an geringer mittzyklischer Schleimbildung und hoher Viskosität des Schleims. b Adäquate mittzyklische Schleimbildung (Quelle: [17]).

eine medikamentöse Ovulationsinduktion zurückgehen, die unabhängig von einer IVF-/ICSI-Therapie erfolgte. Daraus lässt sich ableiten, dass bei einem Großteil dieser Patientinnen auch Clomifen zum Einsatz kam. Dies zeigt anschaulich, dass es sich bei der Clomifengabe zwar um eine vermeintlich einfache Therapie handelt, die jedoch einer sorgfältigen Überwachung bedarf.

Cave: Unter Clomifen ist die erhöhte Inzidenz von Mehrlingsschwangerschaften zu beachten!

Zyklusmonitoring. Jede Form der ovariellen Stimulation bedarf einer entsprechenden Zyklusüberwachung. Hierzu dient die gezielte Ultraschalluntersuchung - ggf. ergänzt durch entsprechende Laboranalytik - zu definierten Zeitpunkten des Stimulationszyklus. Es soll an dieser Stelle noch einmal deutlich herausgestellt werden, dass eine sorgfältige Zyklusüberwachung nicht der Gonadotropinstimulation vorbehalten, sondern ebenso für eine Clomifenstimulation anzuwenden ist. Diesen Grundsatz zu vernachlässigen kann bedeuten, für die Patientin bewusst eine höhergradige Mehrlingsschwangerschaft in Kauf zu nehmen mit allen Konsequenzen, die dies für die betroffene Frau sowie das Paar nach sich zieht.

Eine routinemäßige Ultraschalluntersuchung vor Beginn der Clomifengabe halten wir in aller Regel nicht für erforderlich, sofern eine entsprechende Untersuchung zeitnah vor Indikationsstellung zur Clomifengabe erfolgt ist.

\section{Tipp für die Praxis}

Man sollte den Aufwand des Zyklusmonitorings auf das Notwendige beschränken, da die zeitliche Belastung der Patientin eines der größten Probleme im Rahmen der Kinderwunschtherapie darstellt!

Typischerweise wird 2-3 Tage nach Einnahme der letzten Clomifentablette eine Ultraschalluntersuchung durchgeführt, z. B. am 10.-12. Zyklustag. Diese Untersuchung kann mit einer Hormonanalyse verbunden werden, was wir allerdings nur bei unklarem Ultraschallbefund im Einzelfall für sinnvoll halten. Standardmäßig werden folgende Parameter dokumentiert:

- Anzahl der Follikel (maximaler Durchmesser in einer Ebene) im rechten Ovar

- Anzahl der Follikel (maximaler Durchmesser in einer Ebene) linkes Ovar

- Endometriumdicke/-struktur (homogen/inhomogen)

Sofern der Leitfollikel eine Größe von 17-19 mm erreicht hat, kann man davon ausgehen, dass eine reife Eizelle verfügbar ist.

Es kann dann diskutiert werden, ob gezielt eine Ovulationsinduktion durch hCG-Gabe erfolgt oder ob der spontane weitere Verlauf abgewartet werden soll. Im letzteren Fall sollte aber eine Terminierung der Ovulation z.B. durch LH-Urinsticks erfolgen.

Lässt sich der Follikeldurchmesser deutlich kleiner als $16 \mathrm{~mm}$ darstellen, so wird eine erneute Ultraschalluntersuchung mit einem Abstand von 2-3 Tagen veranlasst, um den weiteren Verlauf abzuwarten. 
Neben der Beurteilung der ovariellen Funktion werden zumeist auch die Dicke des Endometriums und die Homogenität erfasst. Inwieweit die Struktur des Endometriums - trilaminärer Aufbau - sowie die Dicke des Endometriums unter Clomifen jedoch eine Rolle spielen, wird gerade in jüngster Zeit kontrovers diskutiert.

Der Dicke des Endometriums unter Clomifeneinnahme wird lediglich eine marginale Rolle als prognostischer Marker zugewiesen [11].

\section{Tipp für die Praxis}

Sofern sonografisch die Follikelreifung eindeutig darstellbar ist, wird die Hormonanalytik kaum zusätzliche Informationen liefern, die für die Beratung der Patientin und die Steuerung der Stimulation von Belang sind.

Erfolgsrate der Clomifenstimulation. Der Begriff „Erfolgsrate“ wird in der reproduktionsmedizinischen Literatur z.T. sehr unterschiedlich verwendet, so kann als Erfolg der Clomifengabe z. B. der Nachweis einer Ovulation angesehen werden. Hierzu wurde bereits gesagt, dass etwa 50\% der Patientinnen mit zuvor bestehender Anovulation auf $50 \mathrm{mg}$ Clomifen mit einem ovulatorischen Zyklus reagieren. Auch bei Ausschöpfung der maximalen Therapiedosis kommt es letztlich bei etwa 20-25\% der Frauen zu einem Therapieversagen, d. h. auch mit 100-150 mg Clomifen kann keine Ovulation herbeigeführt werden (sog. Clomifenresistenz).

Die erfolgreiche Ovulation stellt nur einen Surrogatparameter dar, denn das Ziel der Behandlung besteht darin, eine Schwangerschaft herbeizuführen.

Die Chance, bei einer Patientin durch Clomifengabe eine Schwangerschaft zu erzielen, hängt neben vielen anderen Faktoren insbesondere auch von der Indikation zur Clomifengabe ab. So konnte gezeigt werden, dass Frauen mit idiopathischer Sterilität nicht mit einer Steigerung der Konzeptionschancen durch Clomifen rechnen können, während Frauen mit Anovulation bzw. Patientinnen mit PCOS nach Clomifengabe eine signifikant erhöhte Schwangerschaftsrate aufwiesen. Die kumulative Schwangerschaftsrate bei Frauen mit Oligomenorrhö betrug nach 6 Zyklen mit Clomifenstimulation ca. 38\% [12].

\section{Metformin}

Metformin ist eines der meistverschriebenen Medikamente und als Standardtherapie des Typ-2-Diabetes etabliert. Metformin verbessert die insulinvermittelte Glukoseaufnahme im Muskel und im Fettgewebe und reduziert die Glukoneogenese der Leber [13]. Unabhängig von diesen günstigen Effekten auf den Stoffwechsel normalisieren sich durch Metformin bei Frauen mit PCOS und den damit assoziierten Störungen der Follikelreifung folgende Parameter:

- Zyklus

- Ovulation

- Hyperandrogenämie

- Hyperandrogenismus

Es zeigt sich unter Metformin eine deutlich bessere Wirkung auf die Akne als auf den Hirsutismus.

Die genannten Effekte lassen sich z.T. auch bei nicht adipösen Patientinnen nachweisen. Interessanterweise sind gerade die gynäkologischen Therapieerfolge der Metformintherapie in den meisten Studien nicht vom Körpergewicht abhängig, sondern fanden sich v.a. bei Patientinnen mit eher niedrigem BMI. Aus diesem Verständnis heraus wurde Anfang der 1990er-Jahre Metformin in die Therapie des PCO-Syndroms eingeführt. Zunächst wurde eine Metformintherapie v.a. diskutiert, wenn Patientinnen mit unerfülltem Kinderwunsch nicht auf eine Clomifenstimulation reagieren. Dabei konnte man sehen, dass durch die Gabe von Metformin nach fehlgeschlagener Clomifenstimulation eine um den Faktor 4 bessere Reaktivität der Ovarien induziert werden konnte.

Tipp für die Praxis

Wegen der in $\mathbf{3 0} \%$ zu erwartenden gastrointestinalen Nebenwirkungen (Meteorismus, Diarrhö) sollte Metformin einschleichend verschrieben werden.

Anwendung von Metformin. Metformin hat eine gute Wirkung bei einem erhöhten BMI $\left(>30 \mathrm{~kg} / \mathrm{m}^{2}\right)$. Bei einem BMI unter $30 \mathrm{~kg} / \mathrm{m}^{2}$ ist zur Ovulationsinduktion Clomifen effektiver als Metformin, jedoch kann diskutiert werden, ob bei Frauen, die auf Clomifen nicht mit einer Follikelreifung reagieren, zusätzlich Metformin zum Einsatz kommen sollte.

Viele Patientinnen setzen Metformin ein, um eine Gewichtsreduktion zu erzielen. Daher soll an dieser Stelle nochmals darauf hingewiesen werden, dass die Therapie mit Metformin allein nicht zu einer Gewichts- 
reduktion führt, sondern dass dies lediglich in Kombination mit einer Lebensstilintervention (Umstellung der Ernährung, ausreichende körperliche Aktivität) funktioniert. Wenn eine Patientin unter einer Therapie mit Metformin über 3-6 Monate kein Gewicht reduziert, sollte die Gabe von Metformin beendet werden, da man in diesen Fällen wohl keinen zusätzlichen Benefit erzielen wird.

\section{Tipp für die Praxis}

Metformin ist nur zur Therapie des Typ-2-Diabetes mellitus zugelassen und muss daher im Falle eines PCO-Syndroms „off Label“, also über ein Privatrezept, verschrieben werden.

Die Dosierung von Metformin richtet sich nach dem BMI:

- Ab einem BMI von $30 \mathrm{~kg} / \mathrm{m}^{2}$ ist eine Dosierung von $2 \times 1000 \mathrm{mg} / \mathrm{d}$ nach einschleichender Dosierung sinnvoll.

- Bis zu einem BMI von $25 \mathrm{~kg} / \mathrm{m}^{2}$ sollten $1500 \mathrm{mg}$ (z.B. $3 \times 500 \mathrm{mg} / \mathrm{d}$ ) ausreichend sein.

- Bei einem BMI von 25-30 kg/m² kann man eine Dosierung von $2 \times 850 \mathrm{mg}$ wählen.

Cave: Kontraindikationen von Metformin: Vor Einleitung der Therapie sind Nieren- und Leberfunktionsstörungen auszuschließen.

Metformin bei eingetretener Schwangerschaft. Metformin ist in Deutschland nicht zum Einsatz in der Schwangerschaft zugelassen. Bereits seit einigen Jahren wird jedoch diskutiert, inwieweit die Gabe von Metformin vor bzw. während der Schwangerschaft in der Lage ist, das Fehlgeburtsrisiko zu senken. Die Datenlage ist bis heute heterogen, die Evidenz der vorliegenden v.a. kleinen und retrospektiven Studien reicht bislang nicht aus, dies eindeutig zu belegen. Deren Zusammenfassung spricht momentan eher gegen diesen erhofften Effekt. Insofern sollte Metformin in der Schwangerschaft abgesetzt werden. Davon sollte nur abgewichen werden, wenn bereits Aborte in der Vorgeschichte aufgetreten sind. In diesem Fall ist die Patientin darüber aufzuklären, dass es keine Evidenz dafür gibt, dass man das Abortgeschehen in der Schwangerschaft beeinflussen kann, dass aber keine Nebenwirkungen für das Kind bzw. unerwünschte Wirkungen für die Schwangere zu erwarten sind und weitere positive Effekte wie die Senkung des Risikos eines Gestationsdiabetes oder anderer Schwangerschaftserkrankungen (Präeklamp- sie) ebenso diskutiert werden, dass aber auch dafür keine ausreichenden Beweise vorliegen.

\section{- Ovarian Drilling}

Schon im vorigen Jahrhundert wurden operative Strategien zur Behandlung des PCOS entwickelt. So galt bis in die 1960er-Jahre die Keilexzision des Ovars als probate therapeutische Option. Heute gibt es für diesen Eingriff definitiv keine Indikation mehr, stattdessen wird im Falle einer chirurgischen Intervention die laparoskopische Punktkoagulation der Ovarialoberfläche vorgenommen. In der internationalen Literatur finden sich die Angaben zu dieser chirurgischen Methode unter

- LOD (laparoscopic ovarian diathermy) oder

- LEROS (laparoscopic electro resection of ovarian surface).

Vorgehen. Es werden verschiedene Vorgehensweisen beschrieben, die sich hinsichtlich der Anzahl der Koagulationspunkte, der Koagulationsstärke sowie der Koagulationszeit unterscheiden. Meist werden pro Ovar 4-10 Koagulationspunkte für jeweils 3-4 Sekunden mit 40 Watt gesetzt [14].

Effektivität. Es werden bei zuvor anovulatorischen Frauen Ovulationsraten von ca. $80 \%$ beschrieben sowie eine deutliche Verbesserung der Zyklizität. Es scheinen v.a. Frauen mit präoperativ deutlich erhöhten LH-Werten von dieser Technik zu profitieren. Die Schwangerschaftsraten innerhalb eines Zeitraums von 5-6 Monaten nach LEROS sind mit dem Ergebnis einer Gonadotropinstimulation über 5-6 Zyklen vergleichbar, jedoch kann der Effekt der LEROS-Operation z.T. über mehrere Jahre anhalten, was einen Vorteil gegenüber konservativen Therapieverfahren darstellt $[15,16]$.

\section{Tipp für die Praxis}

Das Ovarian Drilling kann gewissermaßen als „Ultima Ratio“ zur Behandlung des PCOS bezeichnet werden, sofern konservative Behandlungsmethoden ausscheiden oder ohne Erfolg eingesetzt wurden.

Nachteile. Als potenzieller Nachteil bzw. Risiko muss beachtet werden, dass der Eingriff mit postoperativen Adhäsionen (10-20\% der Fälle) einhergehen kann. Einzelfälle einer postoperativen irreversiblen Ovarialinsuffizienz wurden - bei offensichtlich exzessiver Koagulation - beschrieben. 
Kasuistik zum fallorientierten Lernen

Eine 28-jährige Nulligravida stellt sich mit ihrem Ehemann bei seit 1,5 Jahren bestehendem Kinderwunsch vor.

\section{Anamnese und Klinik}

Die übergewichtige Patientin gibt ein seit Absetzen des oralen Kontrazeptivums unregelmäßiges Regelintervall (35-48 Tage) an. Bereits vor Einnahme des oralen Kontrazeptivums bestand diese Problematik. Zudem seien nun wieder eine vermehrte Behaarung (Gesicht, Bauch, Oberschenkel) sowie auch Akne und Haarausfall aufgetreten. Operationen in der Vorgeschichte bestanden nicht. Eine Chlamydiendiagnostik erfolgte bereits, welche keine Auffälligkeiten ergab. Es werden keine Medikamente eingenommen. Die Mutter der Patientin ist an Diabetes mellitus Typ 2 erkrankt.

Der 32-jährige Partner hat bereits extern eine andrologische Untersuchung durchführen lassen. Hier zeigten sich keine Auffälligkeiten. Das Spermiogramm ergab eine Normozoospermie. Eine regelmäßige Medikation besteht nicht. Nikotin- und regelmäßiger Alkoholkonsum werden verneint.

\section{Arbeitshypothese/ \\ Differenzialdiagnostik}

Primäre Sterilität, V.a. Polyzystisches OvarSyndrom (PCOS). Da die Diagnose eines PCOS den Ausschluss anderer endokriner Erkrankungen voraussetzt, sollte zunächst eine weiterführende Diagnostik erfolgen.

\section{Diagnostik}

Zur Abklärung der Zyklusstörungen Durchführung einer frühfollikulären Kontrolle der Hormonparameter am 3. -5.

Zyklustag: FSH, LH, Östradiol, Testosteron, DHEAS, Prolaktin und TSH.

Diagnose: ovarielle Hyperandrogenämie. In der klinischen Untersuchung zeigt sich in der Tat ein Hirsutismus (Ferriman-Gallwey-Score: 12). Die Vaginalsonografie, welche im Rahmen der gynäkologischen Untersuchung durchgeführt wird, ergibt folgendes Bild (s. Abb. 9).

Zusammen mit der Klinik (chronische Anovulation, Hyperandrogenismus) wer-

$\begin{array}{lll}\text { Hormon } & \text { Laborwert } & \text { Normwert } \\ \text { Östradiol } & 40 \mathrm{pg} / \mathrm{ml} & - \\ \text { FSH } & 4,5 \mathrm{U} / \mathrm{l} & - \\ \text { LH } & 4,0 \mathrm{U} / \mathrm{l} & 0,27-2,5 \mathrm{mU} / \mathrm{l} \\ \text { TSH } & 0,80 \mathrm{mU} / \mathrm{l} & <450 \mathrm{mlE} / \mathrm{l} \\ \text { Prolaktin } & 80 \mathrm{mlE} / \mathrm{l} & 0,06-0,6 \mathrm{ng} / \mathrm{ml} \\ \text { Testosteron } & 0,8 \mathrm{ng} / \mathrm{ml} & 651-3680 \mu \mathrm{g} / \mathrm{ml}\end{array}$

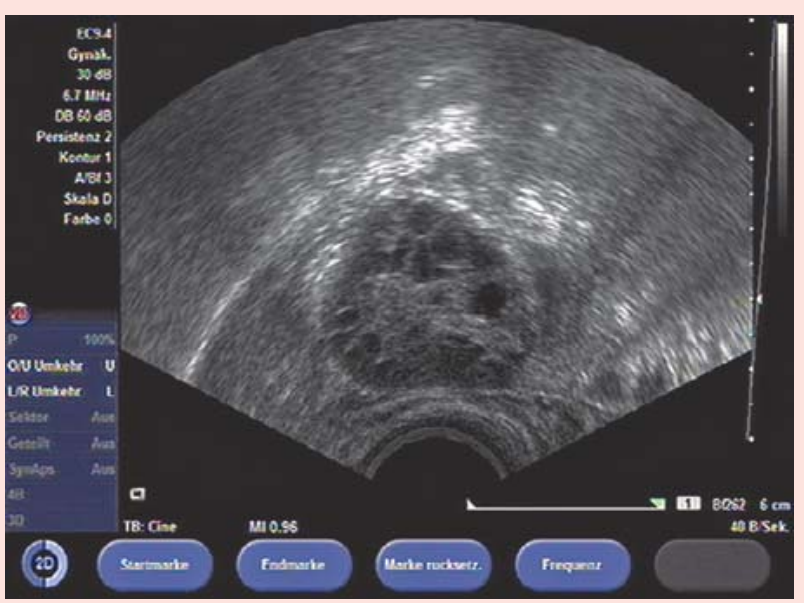

Abb. 9 Ovar mit zahlreichen Antralfollikeln $<10 \mathrm{~mm}$.

den die NIH-Kriterien und auch die Rotterdam-Kriterien für die Definition eines PCOS erfüllt.

Bei Diabetes mellitus Typ 2 in der Familienanamnese und übergewichtiger Patientin (BMI $29 \mathrm{~kg} / \mathrm{m}^{2}$ ) wird die Durchführung eines oralen Glukosetoleranz-Tests besprochen. Hier zeigt sich eine Insulinresistenz (weiterer Anstieg des 2-Stunden-Insulinwertes über den 1-Stunden-Wert hinaus).

\section{Therapie}

Beratung der Patientin hinsichtlich einer Umstellung der Ernährung sowie vermehrte sportliche Aktivität, um eine Gewichtsreduktion zu erreichen, da eine Gewichtsreduktion um $5-10 \%$ des Ausgangsgewichtes bereits zu einer Zyklusregulation führen und die Konzeptionschancen steigern kann. Zusätzlich Start einer Therapie mit Metformin bei nachgewiesener Insulinresistenz (off Label; einschleichende Dosierung). Im Falle der Persistenz der Anovulation erfolgt dann eine Clomifentherapie zur Unterstützung der Follikelreifung (zunächst Start mit 50 mg Clomifen über 5 Tage). Sollte darunter kein ovarielles Ansprechen zu beobachten sein, Steigerung der Clomifendosis. Wenn auch hier keine Follikelreifung erreicht werden kann, Umstellung auf eine Low-Dose-Gonadotropinstimulation. Alternativ kann auch die Durchführung eines Ovarian Drillings besprochen werden.

\section{Weiterführende Betreuung}

Bei dem beschriebenen Paar konnte im Rahmen des 3. Zyklus mit Clomifen eine Schwangerschaft erzielt werden. Aufgrund des erhöhten Schwangerschaftsrisikos (Gestationsdiabetes, Präeklampsie, Frühgeburtlichkeit, höhere perinatale Mortalität) erfolgte eine intensivierte Schwangerschaftsüberwachung. 


\section{Kernaussagen}

Der „unerfüllte Kinderwunsch“ stellt ein häufiges Problem in der gynäkologischen Praxis dar. Die Diagnostik muss sich von vornherein auf beide Partner erstrecken. Dabei müssen auf weiblicher Seite neben Einschränkungen der Tubenfunktion v. a. Störungen der Ovarialfunktion, aber auch allgemeine Endokrinopathien abgeklärt werden. Diagnostik und Therapie von Schilddrüsenerkrankungen sowie der verschiedenen Formen der Hyperandrogenämie können umfassend in der frauenärztlichen Praxis erfolgen - ggf. in enger Zusammenarbeit mit Kollegen anderer Fachgebiete. Die Ovulationsinduktion mit Clomifen - ggf. in Kombination mit Metformin - stellt nach wie vor eine sehr wirksame und in der Praxis gut anwendbare Behandlungsmethode dar.

Führen konservative Behandlungsmöglichkeiten nicht zur Konzeption bzw. zur erfolgreich ausgetragenen Schwangerschaft, so sollte geprüft werden, ob die Indikation für reproduktionsmedizinische Maßnahmen bzw. spezifische Therapieformen (z. B. zur Behandlung habitueller Aborte) besteht. Insbesondere bei solch komplexen Behandlungsverfahren ist die enge und vertrauensvolle Zusammenarbeit zwischen niedergelassenem Frauenarzt und IVF-Zentrum essenziell. Die Autoren hoffen, dass sie mit der vorliegenden Arbeit dazu beitragen können, dass auch zukünftig die Kinderwunschbehandlung einen festen Platz in der gynäkologischen Praxis einnimmt.

\section{Über die Autoren}

\section{Christoph Keck}

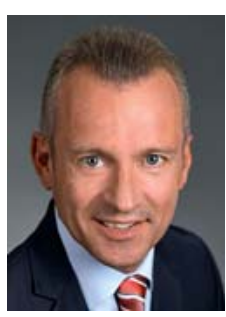

Prof. Dr. med. Studium der Medizin in Münster. Forschungsaufenthalte in Wien, London, Sydney und Melbourne. 1991 Tätigkeit am Institut für Reproduktionsmedizin an der Universität in Münster. 1992-2004 Leitung der Kinderwunschsprechstunde an der Universitätsfrauenklinik Freiburg. 2001 Leitender Oberarzt und Vertreter des Direktors der UniFrauenklinik. Habilitation zum Thema: „Ovarielle Überstimulation“. 2004-2006 Tätigkeit als Global Medical Director in einem international führenden Pharmaunternehmen. 2006 2011 Leitender Arzt eines privaten Instituts für Endokrinologie und Reproduktionsmedizin in Köln. Seit Februar 2011 Leiter des Fachbereichs Gynäkologie der EndokrinologikumGruppe bundesweit. Seit September 2013 Ärztlicher Leiter des Endokrinologikums Hamburg und seit 2014 Ärztlicher Leiter der Sparte „Sprechende Medizin“ amedes Medizinische Dienstleistungen $\mathrm{GmbH}$.

\section{Sabine Elisabeth Segerer}

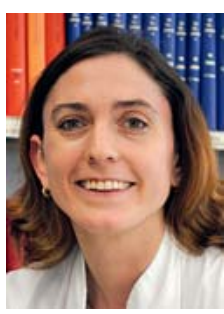

PD Dr. med. Jahrgang 1978. 1998 2005 Studium der Humanmedizin an der Julius-Maximilians-Universität Würzburg und in Vellore (Tamil Nadu, Indien). Dissertation am Institut für Physiologie der Universität Würzburg, 2006 Promotion. 2005-2012 Tätigkeit an der Universitätsfrauenklinik Würz-

burg; 2011 Facharzt-Anerkennung Gynäkologie und Geburtshilfe. 2012 Forschungsaufenthalt an der University of Adelaide, Institute for Reproductive Immunology, 2012 Habilitation zum Thema „Untersuchung des Einflusses von Cytokinen, Chemokinen und hormonellen Faktoren auf Immunzellen der Frühschwangerschaft“. 2013 Schwerpunkt Endokrinologie und Reproduktionsmedizin. Seit 03/2013 Endokrinologikum Hamburg.

\section{Korrespondenzadresse}

Prof. Dr. med. Christoph Keck

Ärztlicher Leiter Endokrinologikum

Lornsenstraße 4-6

22765 Hamburg

E-Mail: Christoph.Keck@endokrinologikum.com 


\section{Literatur}

1 Crosignani PG, Rubin BL. Optimal use of infertility diagnostic tests and treatments. The ESHRE Capri Workshop Group. Hum Reprod 2000; 15: 723-732

2 Stephen EH, Chandra A. Declining estimates of infertility in the United States: 1982 -2002. Fertil Steril 2006; 86: 516-523

3 Mascarenhas MN, Flaxman SR, Boerma T et al. National, regional, and global trends in infertility prevalence since 1990: a systematic analysis of 277 health surveys. PLoS Med 2012; 9: e1001356

4 Li Y, Yang D, Zhang Q. Impact of overweight and underweight on IVF treatment in Chinese women. Gynecol Endocrinol 2010; 26: $416-422$

5 Schliep KC, Mumford SL, Ahrens KA et al. Effect of male and female body mass index on pregnancy and live birth success after in vitro fertilization. Fertil Steril 2015; 103: 388-395

6 Rittenberg V, Sobaleva S, Ahmad A et al. Influence of BMI on risk of miscarriage after single blastocyst transfer. Hum Reprod 2011; 26: $2642-2650$

7 Hamilton JA, Larson AJ, Lower AM et al. Routine use of saline hysterosonography in 500 consecutive, unselected, infertile women. Hum Reprod 1998; 13: 2463 -2473

8 La Marca A, Grisendi V, Griesinger G. How much does AMH really vary in normal women? Int J Endocrinol 2013; 2013: 959487

9 Moran LJ, Brinkworth GD, Norman RJ. Dietary therapy in polycystic ovary syndrome. Semin Reprod Med 2007; 26: 85-92

10 Rostami-Hodjegan A, Lennard MS, Tucker GT et al. Monitoring plasma concentrations to individualize treatment with clomiphene citrate. Fertil Steril 2004; 81: 1187-1193
11 Asante A, Coddington CC, Schenck L et al. Thin endometrial stripe does not affect likelihood of achieving pregnancy in clomiphene citrate/intrauterine insemination cycles. Fertil Steril 2013; 100: 1610-1614.e1

12 Imani B, Eijkemans MJ, te Velde ER et al. A nomogram to predict the probability of live birth after clomiphene citrate induction of ovulation in normogonadotropic oligoamenorrheic infertility. Fertil Steril 2002; 77: $91-97$

13 Tan S, Hahn S, Benson S et al. Metformin improves polycystic ovary syndrome symptoms irrespective of pre-treatment insulin resistance. Eur J Endocrinol 2007; 157: 669-676

14 Seufert R, Gomez R, Skala C et al. Das PCO-Syndrom - aktuelle Aspekte für die Praxis. Frauenheilkunde up2date 2011; 5: 309316

15 Bayram N, van Wely M, Kaaijk EM et al. Using an electrocautery strategy or recombinant follicle stimulation hormone to induce ovulation in polycystic ovary syndrome: randomised controlled trial. BMJ 2004; 328: $192-195$

16 Amer SA, Banu Z, Li TZ et al. Long-term follow-up of patients with polycystic ovary syndrome after laparoscopic ovarian diathermy: endocrine and ultrasonographic outcomes. Human Reprod 2002; 11: $2851-2857$

17 Keck C, Hrsg. Kinderwunsch in der gynäkologischen Praxis. Stuttgart: Thieme; 2014

\section{Interessenkonflikt}

Die Autoren geben an, dass kein Interessenkonflikt besteht. 


\section{CME-Fragen}

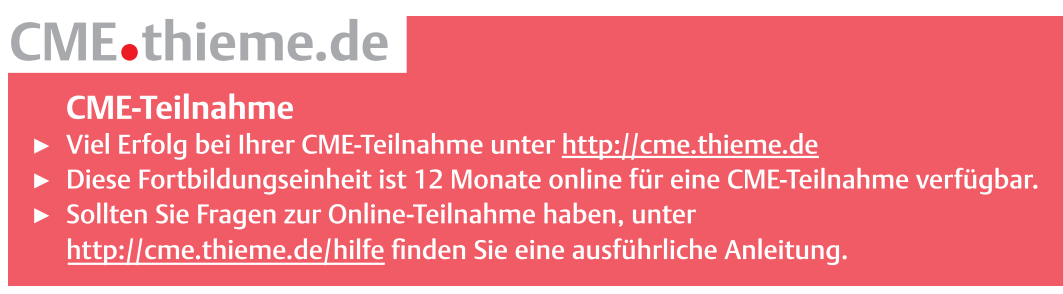

1 Welche Aussage ist richtig?

A Die Geburtenrate in Deutschland steigt seit ca. 10 Jahren kontinuierlich an.

B 2011 gehört zu den geburtenstärksten Jahren.

C Reproduktionsmedizinische Behandlungen tragen signifikant zur Steigerung der Geburtenzahlen in Deutschland bei.

D Fekundabilität beschreibt die Wahrscheinlichkeit des Eintretens einer Schwangerschaft pro Menstruationszyklus.

E Sekundäre Sterilität bedeutet, es liegen Sterilitätsursachen aufseiten des Partners der Patientin vor.

$2 \quad$ Welche Aussage ist falsch?

A Auch bei unerfülltem Kinderwunsch lassen sich bereits durch sorgfältige Anamneseerhebung Hinweise auf mögliche Ursachen gewinnen.

B Bereits im Erstgespräch sollten Informationen von beiden Partnern eingeholt werden.

C Mäßiger Konsum von Alkohol oder Nikotin ist für den Fertilitätsstatus des Paares irrelevant.

D Zyklusstörungen gehören zu den häufigen Symptomen für Fertilitätsstörungen der Frau.

E Deszensusstörungen können als (Teil-)Ursache männlicher Fertilitätsstörungen angesehen werden.

3 Welche Aussage ist richtig?

A Es ist umstritten, ob ein erhöhter BMI sich generell negativ auf die Implantationsrate auswirkt.

B Während Adipositas negative Auswirkungen auf die Fertilität hat, führt ein erniedrigter BMI $\left(<18 \mathrm{~kg} / \mathrm{m}^{2}\right)$ zu einer Verbesserung der Fertilität.

C Die routinemäßige gynäkologische Tastuntersuchung ist bei Frauen mit unerfülltem Kinderwunsch entbehrlich, da sich daraus keine weiterführenden Informationen ableiten lassen.

D Bei Übergewicht kommen die Krankenkassen für die Kosten der Kinderwunschbehandlung nicht auf.

E Bei deutlichem Übergewicht (BMI $>30 \mathrm{~kg} / \mathrm{m}^{2}$ ) muss ein spezifischer Antrag zur Kostenübernahme für die Kinderwunschbehandlung bei den zuständigen Krankenkassen gestellt werden.

$4 \quad$ Welche Aussage ist falsch?

A Nach wie vor gilt die Laparoskopie mit Chromopertubation als „Goldstandard“ zur Abklärung des Tubenfaktors.

B Laparoskopie/Hysteroskopie + Chromopertubation und HSKS haben die gleiche Aussagekraft zur Abklärung der Tubenfunktion.

C Bevor eine operative Abklärung des Tubenfaktors vorgenommen wird, empfiehlt es sich, den Fertilitätsstatus des Partners zu überprüfen.

D Durch Laparoskopie/Hysteroskopie lassen sich Hinweise auf das Vorliegen einer Endometriose gewinnen.

E Die Spezifität der HKSK liegt im Bereich von 70\%.

5 Welche Aussage ist richtig?

A Die Basisuntersuchung zur Erfassung des Hormonstatus sollte typischerweise in der 2. Zyklushälfte durchgeführt werden.

B Bevor weitergehende hormonelle Untersuchungen erfolgen, sollte initial zunächst AMH bestimmt werden.

C Bei niedrigem AMH besteht eine Kontraindikation für die Kinderwunschbehandlung.

D Zur Abklärung des Androgenhaushalts dient in der Basisuntersuchung die Bestimmung von Dihydrotestosteron.

E Prämenstruelles Spotting bzw. Zwischenblutungen können auf ein Lutealphasendefizit hinweisen. 
A AMH wird in Granulosazellen der Primär-, Sekundär- und Antralfollikel gebildet.

B Die AMH-Bestimmung erlaubt eine Abschätzung der ovariellen Reserve.

C Der AMH-Wert gibt die durchschnittliche Eizellqualität an.

D Eine einzelne AMH-Bestimmung ohne spezifische anamnestische Angaben ist wenig aussagekräftig.

E Die AMH-Serumkonzentration ist während des Menstruationszyklus vergleichsweise konstanter als die FSH-Serumkonzentration.

\section{Welche Aussage ist falsch?}

A Die Hyperprolaktinämie gehört zu den häufigsten Ursachen von Zyklusstörungen.

B Psychopharmaka können zu erhöhten Prolaktinkonzentrationen im Serum führen.

C Makroprolaktinome sind wesentlich seltener als Mikroprolaktinome.

D Die Behandlung der Hyperprolaktinämie erfolgt meist durch Gabe von Dopaminagonisten.

E Die Behandlung der Hyperprolaktinämie erfolgt meist chirurgisch durch Entfernung des Prolaktinoms.

\section{$8 \quad$ Welche Aussage ist falsch?}
A Schilddrüsenfunktionsstörungen betreffen Frauen etwa 10-mal häufiger als Männer.
B Ein erhöhter TSH-Wert weist auf eine Hypothyreose hin.
C Bei erniedrigtem TSH-Wert erfolgt die Therapie mit L-Thyroxin oral.
D Eine Hyperthyreose ist vergleichsweise seltener als eine Hypothyreose.
E Im ersten Drittel der Schwangerschaft erfolgt die Behandlung der Hyperthyreose mit Propylthiouracil.

\section{Welche Aussage ist falsch?}
A Wir unterscheiden eine Hyperandrogenämie ovariellen bzw. adrenalen Ursprungs.
B 17-OH-Progesteron gilt als Marker für die adrenale Hyperandrogenämie.
C Die häufigste Ursache der Hyperandrogenämie ist das PCOS.
D Die Behandlung des PCOS erfolgt durch Keilexzision des Ovars.
E Frauen mit PCOS weisen in vielen Fällen eine Insulinresistenz auf.

\section{$10 \quad$ Welche Aussage ist falsch?}
A Clomifen gehört zu den am häufigsten eingesetzten Medikamenten zur Ovulationsinduktion.
B Die Startdosis wird meist mit $50 \mathrm{mg} / \mathrm{d}$ gewählt.
C Das OHSS gehört zu den typischen Komplikationen der Clomifentherapie.
D Bei Clomifenbehandlung kommt es zu einem Anstieg des Risikos für Mehrlingsgraviditäten.
E Die sorgfältige Überwachung einer Clomifenstimulation durch Ultraschall und ggf. Hormonanalysen ist obligat. 